Gentieu, T., Catapano, A., Jumel, J. and Broughton, J. (2017) 'Computational modelling of particulate-reinforced materials up to high volume fractions: Linear elastic homogenisation', Proceedings of the Institution of Mechanical Engineers, Part L: Journal of Materials: Design and Applications

DOI: https://doi.org/10.1177/1464420717707227

This document is the authors' Accepted Manuscript.

License: https://creativecommons.org/licenses/by-nc-nd/4.0

Available from RADAR: https://radar.brookes.ac.uk/radar/items/68c35f75-02f3-480d-8078-7ab9665ce96e/1/

Copyright $(\subseteq$ and Moral Rights are retained by the author(s) and/ or other copyright owners unless otherwise waved in a license stated or linked to above. A copy can be downloaded for personal non-commercial research or study, without prior permission or charge. This item cannot be reproduced or quoted extensively from without first obtaining permission in writing from the copyright holder(s). The content must not be changed in any way or sold commercially in any format or medium without the formal permission of the copyright holders. 


\title{
Computational modelling of particulate reinforced materials up to high volume fractions: linear elastic homogenisation
}

\author{
Timothée Gentieu ${ }^{1,2}$, Anita Catapano ${ }^{3}$, Julien Jumel ${ }^{1}$, James Broughton ${ }^{2}$
}

\begin{abstract}
This work focuses on the analysis of the micro and macroscopic mechanical response of particle reinforced composites. A particular attention is paid to the influence of two fundamental design parameters, i.e. the particles shape and their volume fraction (up to very high values ranging from 0 to almost 0.8 ), on the overall mechanical response of the structure as well as on the resulting elastic symmetry of the material. The strain energy-based homogenisation technique of periodic media is here applied to a 2D finite element model of a representative volume element of the composite. Different algorithms are developed to generate, with a good level of accuracy, the real microstructure of the composite material characterised by circular as well as polygonal particles. Moreover, for each studied configuration a link between the geometrical parameters of the microstructure (particles shape, size, distribution and volume fraction) and the size of the representative volume element is also provided in order to properly describe the constitutive behaviour of the composite at the macroscopic scale. The numerical results are compared with analytical models taken from the literature to prove on the one hand the limitations of the analytical approaches and on the other hand the effectiveness of the proposed numerical models.
\end{abstract}

\section{Keywords}

Particle-reinforced composites, Elastic properties, Computational mechanics, Homogenisation, Microstructure

\section{Introduction}

The addition of stiff particles in a bulk matrix is a common practice to obtain a reinforced material stiffer and more resistant than the bulk matrix itself. Such a resulting composite material is used in multiples industries such as automotive, aeronautics, aerospace, and many others. Numerous and very diverse applications have been developed with this kind of composite materials from concrete to metal alloys. Contrary to fibre reinforced composites, particulate reinforced composites do not show strong mechanical principal directions, but, like classical fibre reinforced matrix composites, their design requires a multi-scale approach that implies the determination of mechanical properties of such complex and heterogeneous materials.

Numerous studies have already been carried out to determine experimentally mechanical properties of particles reinforced composites such as stiffness, strength and toughness, as function of different parameters such as particles sizes, volume fractions, shapes, aspect ratios, etc. One of the most complete review on particulate composites can be found in Fu et al. (2008); Nemat-Nasser and Hori (2013). In this study, the authors focus on polymer matrix reinforced composites. Experimental data are gathered and compared to results obtained through the utilisation of simple analytical homogenisation models. One of the main conclusions of this study is that the size of the inclusions has no significant influence on Young's modulus, whilst volume fraction seems to be the most influential of the parameters. These results are in accordance with the experimental ones carried out on silica spherical shaped particle embedded in an epoxy matrix in Adachi et al. (2008);
Ishai and Cohen (1967); Nakamura et al. (1992). All of these works observe that the size of particle has no impact on the elastic moduli of the composite. On the other hand, Spanoudakis and Young (1984) seems to show a slight decrease in Young's modulus for increasing size of particle. However, Spanoudakis and Young (1984) explains this phenomenon by asserting that the experimental procedure, based on flexural tests, is detrimental to bigger particles. All the cited studies focus on composites with spherical shaped particles.

To estimate the elastic properties of heterogeneous materials, multiple homogenisation models have been developed. Some of these models are considered in the present study: Reuss and Voigt's bounds (which considers the two phases respectively in series or in parallel), Hashin and Shtrikman's bounds Hashin and Shtrikman (1963) (which represent the tightest bounds one can get with no microstructural information). The other considered models are based on the pioneering work of Eshelby's inclusion problem Eshelby (1957): Mori-Tanaka Mori and Tanaka (1973) revisited by Beneviste

\footnotetext{
1 Université de Bordeaux, I2M CNRS UMR 5295, F-33400 Talence, France

${ }^{2}$ Oxford Brookes University (OBU), Wheatley Campus, OX331HX, UK ${ }^{3}$ Bordeaux INP, Université de Bordeaux, I2M CNRS UMR 5295, F-33400 Talence, France
}

\section{Corresponding author:}

Anita Catapano, Bordeaux INP, Université de Bordeaux, I2M CNRS UMR 5295, F-33400 Talence, France, tel: +33 540006220.

Email: anita.catapano@bordeaux-inp.fr 
Benveniste (1987) where inclusions are clearly considered embedded in the matrix, the self-consistent scheme Budiansky (1965); Hill (1965) where particles and matrix are embedded in the homogenised material, and Lielens' model Lielens et al. (1998) which is based on a non trivial interpolation between Mori-Tanaka and inverse Mori-Tanaka model (matrix embedded in the inclusions). All the models derived from Eshelby's theory take into account the volume fraction and the shape of the particles. One can also note that the Mori-Tanaka homogenisation scheme gives exactly the same results as the Hashin and Shtrikman's lower bound for a biphasic material. These models are called uniform fields models because they do not account for local variations in the strain and stress fields. This is a significant limitation regarding these models, especially when local nonlinear phenomena or complex microstructural morphologies are introduced.

To overcome these difficulties, several authors have developed a numerical tool where they consider the evolution of simple pattern like a single inclusion in a matrix. This unit cell approach is based on the assumption that the material microstructure is fully periodic. However, observing the microstructure of particle reinforced composite materials, such an assumption seems to be very unrealistic because most of these materials show highly random microstructures. This microstructural randomness can be represented in a better way with Representative Volume Elements (RVE) approach, where a synthetic random microstructure is generated. The definition and the size of the RVE is a complicated question, as discussed in Kanit et al. (2003). Once the size of the RVE is defined (which has to be sufficiently large to be representative of the macroscopic behaviour of the real material) and the microstrcuture randomly generated, three types of boundary conditions can be imposed: Kinematic Uniform Boundary Conditions (KUBC, where a uniform displacement is applied to the boundaries of the RVE), Static Uniform Boundary Conditions (SUBC, where a uniform traction is applied on the boundaries of the RVE), and Periodic Boundary Conditions ( $\mathrm{PBC}$, where a relative displacement between all the points on opposing sides of the RVE is imposed). Then the problem is solved numerically, using methods such as Finite Elements (FE) or Fast Fourier Transformation (FFT). As explained in Kanit et al. (2003), the KUBC tends to overestimate the homogenised elastic moduli whilst the SUBC tends to underestimate it. The results of the PBC lie somewhere between these two bounds and the periodic boundary conditions seem to be the best compromise.

Multiples studies based on a numerical approach have already been carried out to obtain numerically the homogenised elastic moduli of particle reinforced composites. Synthetic microstructures with spherical or ellipsoidal particles are created and then the mechanical problem is resolved with a FE analysis in Segurado and Llorca (2002); Kari et al. (2007); Singh et al. (2015) or with FFT method in Ghossein and Lévesque (2012). These works show that they are able to evaluate the elastic moduli up to a maximum $60 \%$ of particles volume fraction.

The aim of the present work is to develop numerical FE-based models capable of reproducing a composite microstructure which is representative of the one observed on real materials in order to better understand its mechanical response at both micro- and macro-scale levels. Subsequently, these models will be used to perform numerical analyses in order to calculate the full set of elastic properties of the material. With such protocol, we will be able to determine the effective elastic properties of the composite as well as the degree of anisotropy of the equivalent homogeneous material at the macro-scale. In particular, three different algorithms are developed in order to reproduce microstructures characterised by several particle shapes as well as microstructures having high volume fractions of particles up to $80 \%$, which is a very difficult task to achieve. Indeed, a particular attention is paid to the influence of two fundamental design parameters on the mechanical response of the composite: the particles shape and their volume fraction. In addition, to prove the effectiveness of the proposed FE models the sensitivity to particles size and distribution over the RVE as well as the size of the RVE is studied too and the results are compared with analytical ones taken from literature. For sake of clarity and reduced calculation time, in the present study, the results and analyses are presented regarding only the $2 \mathrm{D}$ case. The computational model is implemented under plain strain assumption that is characteristic of elongated structures loaded in the transverse directions. This approach can be explained by asserting that according to Böhm and Han (2001); Singh et al. (2015), the authors are aware that 3D models are clearly superior to 2D ones, howevewer planar models give accurate trends of results and a good idea of the behaviour of a microstructure. The paper is organised as follows: the numerical homogenisation strategy is explained in Section, then Section focuses on the algorithms developed to generate the RVE, Section describes the homogenisation procedure implemented for this study, and finally the results of the homogenisation procedure are discussed in Section .

\section{FE-based homogenisation strategy}

In the past, several analytical, numerical and experimental techniques have been developed in order to determine the effective properties of composite materials as a function of geometric and material properties of both the inclusions and the matrix. Each method presents a certain level of sophistication. Contrary to analytical theories, numericalbased techniques such as FE methods, do not make use of simplifying assumptions and are not expensive as the experimental tests. In addition, depending on the level of refinement of the model, they can lead to realistic solutions in terms of the elastic response of the structure. Therefore we have chosen a FE-based homogenisation strategy for predicting the elastic properties of a particulate reinforced composite.

Unlike periodic structures, such as cellular solids, particlefilled composites do not show a repetitive unit that neatly reproduces the microstructure of the composite. In these materials the particles, in fact, are more or less randomly distributed and the resulting microstructure is a matrix containing a certain volume fraction of inclusions inordinately distributed. Therefore, we will apply the homogenisation method at the micro-scale (the scale of the particles) not, as usually done, on a repetitive volume 
element but on a RVE able to take into account within the elastic response of the structure also the random distribution of the inclusions and their shape. Then, the actual heterogeneous structure, at the macro-scale level, will be replaced by an equivalent homogeneous medium characterised by the elastic properties determined during the homogenisation phase. About the degree of symmetry of the homogenised structure, no assumptions are made, and all of the components of stiffness tensors are calculated. Thus, only in a second time, through the analysis of the stiffness tensor components, the type and degree of elastic symmetry of the equivalent homogeneous medium is determined. Only after this analysis, we are able to state that the equivalent medium is actually isotropic, orthotropic, etc.

The key points of this study are that the numerical procedure allows to represent in a realistic manner random distributions of particles with different shapes up to a high volume fraction through a precise definition of the RVE and that no assumptions are made about the elastic symmetries of the equivalent homogenised structure. The basic assumptions made to evaluate the elastic response of the model and, hence, to determine the effective properties are:

1. linear, elastic behaviour for the materials of the matrix and particles;

2. perfect bonding for the wall-to-wall contact between matrix and particles.

An outline of the numerical homogenisation procedure developed in the framework of this study is presented in Fig. 1. The first step concerns the generation of the microstructure of the a RVE (characterised by imposed shapes, sizes and volume fraction of the particles). The algorithms that have been developed to generate the RVE are described in Sec. . During the second step of the strategy, the FE-based homogenisation is performed on the RVE. Finally, a homogenised stiffness tensor is extracted, that gives the effective elastic moduli of a particles reinforced composite considered as an equivalent homogeneous material. The homogenisation procedure is detailed thereafter.

\section{Generation of the microstructure}

To generate the RVE for a particulate reinforced composite, multiple strategies can be used. The first characteristic to take into account is the shape of the particles. For this study, carried out in 2D, two shapes of particles are used: circular and polygonal particles. We also remind that the second objective of this work is to evaluate the response of highly filled composite materials, therefore the microstructure of the RVE has to reach high volume fraction of particles. Two families of sphere packing algorithms can be distinguished: the dynamic and the constructive ones. Concerning the dynamic algorithms, the positions and/or sizes of particles as well as the interactions between them are updated continuously during the iterations of the algorithms. For this reason, they are usually time-consuming. For example, moving and shrinking or growing algorithms (Lubachevsky and Stillinger (1990); Lubachevsky et al. (1991)) are well known dynamic algorithms. On the other hand, for the constructive algorithms, the position and the size of each particle is defined sequentially and, when a particle is placed, its position is recorded and will not be updated. The most intuitive algorithm to generate a microstructure is the Random Sequential Adsorption (RSA) algorithm, where the location of each particle is randomly defined Torquato (1991). However, this algorithm can't achieve high volume fraction (up to a maximal value of 0.3 ). That is the reason why several studies (Segurado and Llorca (2002); Singh et al. (2015); Kari et al. (2007); Ghossein and Lévesque (2012)) use a an improved version of the RSA algorithm. In any case, even when using an improved version of the RSA algorithm, it is not possible to achieve volume fractions higher than about 0.6.

\section{Dropping and Rolling algorithm}

In the present article, the authors decided to use a dropping and rolling algorithm based on the work of Visscher and Bolsterli (1972) and Shi and Zhang (2006). This method is able to generate denser microstructures than the previously cited constructive algorithms. The steps characterising the algorithm (see Fig. 2) developed in the framework of the present study are described thereafter:

1. A first sphere is dropped from a random position on the upper side of the frame;

2. the sphere falls (incremental vertical displacement) until it touches the floor (bottom of the RVE) and its final position is recorded;

3. a new sphere is dropped from another random position;

4. during the fall, two possibilities appears:

(a) no previous sphere is under the falling one and the latter goes straight to the floor;

(b) there is a contact between the falling sphere and already placed ones $\Rightarrow$ the new sphere rolls on the previous ones until it reaches an equilibrium position: either hitting the floor or on two existing spheres;

5. repeat the process from step 3 item until the box is filled with spheres.

Roughly speaking, this procedure is very similar to grains of sand flowing into a box. The advantage of such an algorithm is that the microstructure generated can reach high volume fraction of inclusions in a reasonable time. Moreover, a radii distribution can be defined (either discrete or continuous), unlike most dynamic methods. The final volume fraction attained is controlled via a parameter representing the interinclusion minimal distance. Two boundaries were developed for the dropping and rolling algorithm. The first one is called "rigid wall" where the disks cannot bypass those walls (see Fig. 5 (a)). The second one is called "soft wall": the disks are allow to bypass the walls and they reappear on the opposite side of the frame to ensure periodicity of the microstructure (see Fig. 5 (b)). The soft wall conditions also avoid non homogeneous particles distribution near the boundaries of the RVE and reach higher volume fractions.

\section{Voronoi tesselation microstructure algorithm}

The spherical shape of the inclusions in a particulate reinforced composite is not always a realistic assumption. In some materials, the inclusions show multiples facets 
and sharp angles. To deal with this kind of microstructure, an algorithm based on a Voronoi tesselation has been implemented, see Fig. 3. The steps charactering such an algorithm are described thereafter:

1. $\mathrm{N}$ seeds (points) are randomly generated in the frame delimited by RVE sides. A loop can be implemented to recreate seeds that are to close to other ones;

2. this frame (with seeds) is duplicated 8 times and translated all around the frame (top, bottom, right, left and in diagonals). This step ensures that when the growth step of the grains occurs, the periodicity of the boundaries is respected;

3. the edges of all the cells are created using a Delaunay triangulation (the Voronoi diagram being the dual graph of this triangulation); the Matlab functions delaunayTriangulation and voronoiDiagram are used here. In fact, this process gives the same results as circles growing from each seed. The boundaries are created when two points from two circles are in contact;

4. a reduction factor is applied to all the cells so that their edges are no longer in contact to create the matrix phase. The advantage of such a reduction is that the volume fraction of particles is exactly determined by this factor.

A typical microstructure obtained with this algorithm is depicted in Fig. 5 (c).

\section{Polygonal microstructure from Dropping and Rolling algorithm}

The dropping and rolling algorithm was modified to include polygonal particles, which may be more representative of the microstructure. The steps charactering such an algorithm (see Fig. 4) are described thereafter:

1. A microstrcuture is created using the dropping and rolling algorithm;

2. each circular particle is transformed into a polygonal inscribed in the circle:

(a) the number of vertices of each polygon $N_{\text {angles }}$ is randomly generated between 5 and 8;

(b) for each vertex $n$ (from 1 to $N_{\text {angles }}$ ), a number $\theta_{n}$ between $(2 \pi) /(n-1)$ and $(2 \pi) /(n)$ is randomly generated. The use of intervals is included to avoid obtaining polygons that are too distorted;

(c) the coordinates of each vertex of the polygon is the intersection of a line going from the center of the circular particle and forming and angle $\theta_{n}$ from horizontal and the circle:

$$
\left\{\begin{array}{l}
x_{n}=x_{0}+R \cos \left(\theta_{n}\right) \\
y_{n}=y_{0}+R \sin \left(\theta_{n}\right)
\end{array}\right.
$$

where $\left\{x_{0} ; y_{0}\right\}$ are the coordinates of the circular particle's center and $R$ is its radius.

This algorithm has been implemented using either rigid or soft wall boundary conditions. In order to avoid boundary effects, only soft wall boundary types will be used in the present study. The resulting microstructure is represented in Fig. 5 (d).

\section{Geometrical capacities of these algorithms}

Finally, three different models were generated based on the algorithms described beforehand: Dropping and Rolling algorithm with rigid walls or soft walls, the Voronoi tesselation-based algorithm, and Dropping and Rolling with polygonal particles. The geometrical capacities of these algorithms are shown in Table 1.

The first algorithm generates microstructures with circular particles whereas the latter two generate polygonal particles. A parameter $\epsilon$, representing the inter-particle minimal distance, is used in the Dropping and Rolling algorithms to set up the volume fraction. However, the volume fraction is for this reason unknown a priori and calculated once the microstructure has been generated. One can clearly see that the soft wall boundary condition allows higher volume fractions of particles than the rigid wall conditions. Also, the creation of inscribed polygons reduces the maximum volume fraction. One of the strengths of the Voronoi tesselationbased algorithm is that the number of particles and their volume fraction are inputs to the model. Unlike the Dropping and Rolling algorithms, the particles size distribution cannot be employed with this algorithm.

\section{The 2D numerical-based homogenisation procedure}

The effective properties of the particles reinforced composite are determined by using the strain energy homogenisation technique Catapano and Jumel (2015): repetitive periodic structures are used to approximate the effective properties of the composite at the macro scale. This technique is based on the assumption that the periodic heterogeneous structure undergo the same global deformation as the homogeneous solid, so that they have, hence the same strain energy. The geometry of the RVE is generated with the algorithms described in Section .

\section{Periodic Boundary Conditions (PBC)}

Periodic boundary conditions are applied to the square cell faces in order to ensure continuity of the displacement, stress and strain fields. The displacement of corresponding nodes on opposite faces are coupled. Let us denote the two directions of the plane as 1 and 2. The periodic boundary conditions can be expressed as a function of the displacement vector $\underline{u}$ as:

$$
\left\{\begin{array}{l}
\underline{u}(0, y)-\underline{u}(L, y)=\underline{u_{1}}, \\
\underline{u}(x, 0)-\underline{u}(x, L)=\underline{u_{2}},
\end{array}\right.
$$

where $L$ is the length of both sides of the RVE. Then, to obtain three different loading cases in the plane (tensile loading along 1-direction, tensile loading along 2-direction, and shear in the 12-plane), three displacement combinations are imposed as presented in Tab. 2.

The global strain in the RVE is then calculated thanks to the following equation:

$$
\bar{\epsilon}_{11}=\frac{u_{1}}{L}, \bar{\epsilon}_{22}=\frac{u_{2}}{L}, \bar{\epsilon}_{12}=\frac{u_{1}+u_{2}}{L} .
$$

Once the linear elastic problem is solved, we can have access to the global averaged stress, which corresponds to an area 
average on the RVE surface, computed as follows:

$$
\bar{\sigma}_{\alpha}=\frac{1}{S_{R V E}} \int_{S_{R V E}} \sigma_{\alpha} \mathrm{d} S, \alpha=11,22,12 .
$$

\section{Reconstruction of the stiffness tensor}

Under plane strain assumption, Hooke's law is written as follows:

$$
\left\{\begin{array}{c}
\bar{\sigma}_{11} \\
\bar{\sigma}_{22} \\
\bar{\sigma}_{12}
\end{array}\right\}=\left[\begin{array}{lll}
C_{1111} & C_{1122} & C_{1112} \\
C_{1122} & C_{222} & C_{2212} \\
C_{1112} & C_{2212} & C_{1212}
\end{array}\right]\left\{\begin{array}{c}
\bar{\epsilon}_{11} \\
\bar{\epsilon}_{22} \\
2 \bar{\epsilon}_{12}
\end{array}\right\} .
$$

Three loading cases are necessary to determine all the components of the effective stiffness tensor in 2D (6 loading cases are required in 3D): uniaxial tension along the 1 -axis and 2-axis and shear in the 12-plane. To reconstruct directly the columns of the effective stiffness tensor, we want to obtain a single non-zero value in the strain tensor $\left(\epsilon_{11}\right.$ in the case of tension along 1-axis, $\epsilon_{22}$ in the case of tension along 2-axis, $\epsilon_{12}$ in the case of the shear in 12-plane). Fig. 6 represents the displacement field of each loading case. From each loading case, the stress and strain fields are determined thanks to eqs. and, and then a column of the stiffness tensor is then calculated using the following relationships (eqs. 6 , $7,8)$ :

$$
\begin{gathered}
\left\{\begin{array}{l}
\bar{\sigma}_{11} \\
\bar{\sigma}_{22} \\
\bar{\sigma}_{12}^{-}
\end{array}\right\}=\left[\begin{array}{lll}
C_{1111} & * & * \\
C_{1122} & * & * \\
C_{1112} & * & *
\end{array}\right]\left\{\begin{array}{c}
\bar{\epsilon}_{11} \\
0 \\
0
\end{array}\right\}, \\
\left\{\begin{array}{l}
\bar{\sigma}_{11} \\
\bar{\sigma}_{22} \\
\bar{\sigma}_{12}
\end{array}\right\}=\left[\begin{array}{lll}
* & C_{1122} & * \\
* & C_{222} & * \\
* & C_{2212} & *
\end{array}\right]\left\{\begin{array}{c}
0 \\
\bar{\epsilon}_{22} \\
0
\end{array}\right\}, \\
\left\{\begin{array}{c}
\bar{\sigma}_{11} \\
\bar{\sigma}_{22} \\
\bar{\sigma}_{12}
\end{array}\right\}=\left[\begin{array}{lll}
* & * & C_{1112} \\
* & * & C_{2212} \\
* & * & C_{1212}
\end{array}\right]\left\{\begin{array}{c}
0 \\
0 \\
2 \bar{\epsilon}_{12}
\end{array}\right\}
\end{gathered}
$$

\section{The 2D FE model}

The finite element analysis is carried out using the commercial FE code ANSYS ${ }^{\circledR}$. The geometry of the RVE, imported in ANSYS $^{\circledR}$, is meshed with plane quadratic triangular elements (PLANE183, 6 nodes triangular elements) having two Degrees Of Freedom (DOFs) per node, i.e. the translations in the nodal 1 and 2 directions. The plain strain option is activated for these elements. A preliminary study allows to ensure that the results are mesh-independent with a mean elements size 100 times smaller than the length of the RVE.

\section{Results and discussion}

The numerical tool described beforehand is used to evaluate the micro as well as the macroscopic behaviour of a particle reinforced composite, as a function of: the elastic moduli of the phases and the volume fraction, size, and shape of inclusions. Three different RVE generation algorithms have been utilised and the results obtained from these types of microstructures will now be compared. The elastic properties of the phases of the composite used for this study are depicted in Table 3. The average size of particles obtained using the algorithms detailed beforehand ranges from $30 \mu \mathrm{m}$ to $100 \mu \mathrm{m}$.

\section{Size of the RVE and its mechanical symmetries}

Before performing the homogenisation, the first task is to determine the minimum size of the RVE able to give a proper representation of the macroscopic response of the composite material. This study is based on both the evolution of the elatic properties scatter and the one of mechanical symmetries as function of the size of the RVE. This is done for a fixed volume fraction of $0.40( \pm 0.005)$. For each algorithm, 20 microstructures are generated for different sizes of RVE (the length of the square side ranging from $0.4 \mathrm{~mm}$ to $3 \mathrm{~mm}$ ). The average for each 20 microstructures and the corresponding standard deviation are represented in Fig. 7. One can observe that under a $1 \mathrm{~mm}$-size RVE, the average values and the standard deviation are not acceptable (the deviation is higher than a maximum tolerance value equal to $1 \%$ ). A size of $1.5 \mathrm{~mm}$ seems to be the best compromise between accuracy and computational time. This size corresponds to approximately 60 particles.

The different algorithms will now be compared according to the mechanical symmetries of the RVE they generate. As well, in these analyses, the dependency of the RVE size is highlighted. This study represents an evolution of the one carried out in Catapano and Jumel (2015) for particulate reinforced adhesives. In particular, in Catapano and Jumel (2015) only circular shaped inclusions have been considered and the analysis on the elastic symmetries of the equivalent homogeneous medium showed an interesting result: particle reinforced composites characterised by circular inclusions can show, in general, an isotropic or at least a cubic elastic symmetry. In the present work, due to the presence of different particles shape, the full set of the components of the stiffness matrix of the RVE is evaluated. Fig. 8 represents the ratio of the tensile elastic moduli in the 2-direction over the 1-direction. The average and the standard deviation are depicted for 20 microstructures and for different volume fractions. As for the size of the RVE study, the variation in results seems to be steady for a RVE size of $1.5 \mathrm{~mm}$. All the algorithms converge to a ratio of 1 , except the dropping and rolling with rigid wall boundaries. In this case, it seems to create a slight orthotropy between the vertical and horizontal directions.

The component $C_{2212}$ of the stiffness tensor should be equal to 0 for at least orthotropic materials (as for most of the materials with lower symmetries). Fig. 9 represents the ratio of $C_{2212}$ over $C_{1111}$ for the dropping and rolling algorithm. We see that for a RVE size of $1.5 \mathrm{~mm}$, the variation of the component $C_{2212}$ is lower than $0.5 \%$ of $C_{1111}$, which falls within the fixed tolerance. Same results are observed for all the other algorithms and also concerning the component $C_{1112}$ of the stiffness matrix.

The isotropy of the concerned microstructures is validated using the shear component $C_{1212}$ of the stiffness tensor. In this case, $C_{1212}$ must be equal to $\left(C_{1111}-C_{1122}\right) / 2$ for isotropic materials. The evolution of the ratio of these components is depicted in Fig. 10. For isotropic microstructures, the ratio should tend to 1 . For the three microstructures based on the dropping and rolling algorithm, 
this ratio tends to a value ranging between 1.03 and 1.05. Although the difference is very low (less than a $5 \%$ difference between $C_{1212}$ and $\left.\left(C_{1111}-C_{1122}\right) / 2\right)$, this means that the microstructures generated from dropping and rolling are slightly cubicle and not completely isotropic. The Voronoi tesselation algorithm gives fully isotropic microstructures as the ratio tends to 1 .

\section{Comparisons with analytical homogenisation models}

The results obtained with this numerical procedure can be compared to those obtained using the analytical homogenisation schemes. To fit with the numerical results obtained under plane strain assumption, the homogenisation schemes presented hereafter are expressed with transversely isotropic structures, a matrix with infinite circular cylinders. The Fig. 11 represents the evolution of the component $C_{1111}$ of the stiffness tensor against the volume fraction of particles for all the analytical homogenisation schemes used in this study: Voigt and Reuss bounds, Hashin-Shtrikman bounds, Mori-Tanaka scheme, Self Consistent Scheme and Lielen's model. The computational results are compared to these analytical models (Fig. 13 (a) for low volume fractions ranging from 0 to 0.5 and (b) for high volume fraction ranging from 0.5 to 0.9 ). The comparison is carried out on a volume fraction range between 0 and 0.9 , even if the dropping and rolling algorithm is not able of producing volume fractions higher than 0.75 . Over the whole range of volume fractions, the results lie between the HashinShtrikman bounds.

For low to moderate volume fraction (0 to 0.4$)$, the elastic moduli given by all the microstructures are very similar and are very close to the lower Hashin-Shtrikman bounds. Below a volume fraction around 0.5 , the results start to differ for the different microstructure types. Similar trends are observed for most of the analytical homogenisation models. The two boundary types (rigid wall and soft wall) give similar homogenised elastic moduli. A difference might appear when nonlinearities are taken into account, because the distribution of particles is less homogeneous due, to boundary effects in the case of rigid wall condition. The dropping and rolling algorithm with polygonal particles also seems to provide similar trends (differences lower than $10 \%$ ). In the case of polygonal particles, we observe slightly stiffer equivalent homogeneous properties. The best analytical homogenisation model to fit these data is Lielen's model, as cited by Klusemann and Svendsen (2010) and Ghossein and Lévesque (2012). The microstructures given by the Voronoi tesselation algorithm give lower homogenised elastic moduli, close to Hashin-Shtrikman lower bounds. This could be due to the fact that, with this algorithm, the sides of the polygons are aligned with respect to that of its neighbours. To picture this difference with the other algorithms, Fig. 12 represents the local stress $\sigma_{11}$ in the matrix of the RVE for a dropping and rolling microstructure and that of the Voronoi models. We can observe that some paths are completely unloaded in the case of the Voronoi microstructure.

The same analysis is made for the component $C_{1212}$ of the stiffness tensor. This component represents the behaviour of the material under shear loading. A comparison between analytical models and numerical results is depicted in Fig. 13 (a) for low volume fractions and in Fig. 13 (b) for higher volume fractions. The numerical results also lie between the Hasin-Shtrikman bounds. The scatter between the different models seems to be a little bit higher than for the $C_{1111}$ component. The same comparisons between analytical models and numerical results can be made, i.e. the results are very similar from low to moderate fractions and they start to differ for higher volume fraction (from $\phi=0.5$ ). Moreover, the Lielens model seems to be the closest to the results from the dropping and rolling RVE whilst the Voronoi tesselation RVE are closer to the lower Hashin-Shtrikman bound. The Voronoi tesselation microstructure shows this time a behaviour above the Hashin-Shtrikman lower bound. Similarly, the dropping and rolling RVE are slightly above Lielens model for this component, especially with polygonal particles. For the dropping and rolling models, this may be explained by the slightly cubicle behaviour that has been observed. Fig. 14 shows the local stress fields $\sigma_{12}$ in the case of dropping and rolling microstructure type with circular particles Fig. 14 (a) and in the case of Voronoi type microstructure Fig. 14 (b). Noticeably higher stress concentrations can be observed for the Voronoi RVE, localised at the angles of the particles. Multiples almost circular unloaded regions are visible between particles in the case of the dropping and rolling RVE, a phenomenon less important on the Voronoi RVE.

\section{Conclusions}

A computational procedure has been implemented to analyse the micro as well as the macroscopic response of particle reinforced composites. A particular attention is paid to the evaluation of the stiffness properties and of the degree of anisotropy characterising the equivalent homogeneous material. Different microstructures characterised by several parameters (particles shape, size and volume fraction) have been analysed. Moreover, for each configuration the minimal size of the RVE for which its behaviour effectively represents the macroscopic response of the composite material has been determined. To generate an accurate microstructure of the RVE three different algorithms have been developed. The first one generates circular particles while the rest generate polygonal shapes. The main results to be highlighted are as follows:

1. The dropping and rolling algorithm appears to be a good model to generate microstructures with circular inclusions up to high volume fractions $(0.75)$, in particular soft wall boundary types are useful to reach higher volume fraction than rigid wall ones.

2. The modified version of the dropping and rolling algorithm via the introduction of polygons (inscribed in the original circles) strongly reduces the highest volume fraction reached. This algorithm, though, generates microstructures that are slightly cubic instead of fully isotropic.

3. The effective elastic moduli obtained using the two types of boundary conditions (i.e. the rigid and the soft wall one) are however very close. A slight difference might however appear when considering 
non-linearities, due to different particles distributions next to the boundaries of the RVE.

4. All the numerical results obtained with the proposed models are close to those given by the analytical models taken from literature just for low to moderate values of the volume fraction (between 0 and 0.4). Whereas, major differences appear at higher values of volume fraction (above 0.6 ). This aspect is strictly related to the interaction (in terms of local stress field) between the inclusions which is more pronounced at high volume fractions than lower ones.

5. Although, the Voronoi-based algorithm has the advantage of generating microstructures with fixed volume fraction and a fixed number of particles, the equivalent stiffness of the resulting composite material is lower than the one belonging to microstructures obtained using the dropping and rolling algorithm (next to the lower Hashin-Shtrikman and Mori-Tanaka bounds). This could be due to the very special microstructure generated with this algorithm, whereby the sides of each polygon are parallel to those of its neighbouring polygons. This orderly geometry causes several continuous stress flows in the matrix from one side to the opposite one of the RVE; in this way the matrix results to be the most charged phase of the composite while the inclusions give only a secondary contribution to the stiffness of the composite.

Finally, even if the shape of the particles seems to have a small effect on the equivalent elastic properties of a particulate composite, it might become much more significant when considering non-linearities (debonding of the particles and matrix plasticity or failure) due to different stress concentrations around these particles. Research is ongoing on these aspects.

\section{Acknowledgements}

DGA of France, supporting the first author through Allocation de thèse 2015, is gratefully acknowledged.

\section{References}

\section{References}

Adachi T, Osaki M, Araki W and Kwon S (2008) Fracture toughness of nano-and micro-spherical silica-particle-filled epoxy composites. Acta Materialia 56(9): 2101-2109.

Benveniste Y (1987) A new approach to the application of moritanaka's theory in composite materials. Mechanics of Materials 6: $147-157$.

Böhm HJ and Han W (2001) Comparisons between threedimensional and two-dimensional multi-particle unit cell models for particle reinforced metal matrix composites. Modelling and Simulation in Materials Science and Engineering 9(2): 4765.

Budiansky B (1965) On the elastic moduli of some heterogeneous materials. Journal of the Mechanics and Physics of Solids 13(4): 223-227.
Catapano A and Jumel J (2015) A numerical approach for determining the effective elastic symmetries of particulatepolymer composites. Composites Part B: Engineering 78: 227243.

Eshelby JD (1957) The determination of the elastic field of an ellipsoidal inclusion, and related problems. In: Proceedings of the Royal Society of London A: Mathematical, Physical and Engineering Sciences, volume 241. The Royal Society, pp. 376-396.

Fu S, Feng X, Lauke B and Mai Y (2008) Effects of particle size, particle/matrix interface adhesion and particle loading on mechanical properties of particulate-polymer composites. Composites Part B: Engineering 39(6): 933-961.

Ghossein E and Lévesque M (2012) A fully automated numerical tool for a comprehensive validation of homogenization models and its application to spherical particles reinforced composites. International Journal of Solids and structures 49(11): 13871398.

Hashin Z and Shtrikman S (1963) A variational approach to the theory of the elastic behaviour of multiphase materials. $J$. Mech. Phys Solids 11: 127-140.

Hill R (1965) A self-consistent mechanics of composite materials. Journal of the Mechanics and Physics of Solids 13(4): 213222.

Ishai O and Cohen L (1967) Elastic properties of filled and porous epoxy composites. International Journal of Mechanical Sciences 9(8): 539-546.

Kanit T, Forest S, Galliet I, Mounoury V and Jeulin D (2003) Determination of the size of the representative volume element for random composites: statistical and numerical approach. International Journal of solids and structures 40(13): 36473679.

Kari S, Berger H, Rodriguez-Ramos R and Gabbert U (2007) Computational evaluation of effective material properties of composites reinforced by randomly distributed spherical particles. Composite Structures 77(2): 223-231.

Klusemann B and Svendsen B (2010) Homogenization methods for multi-phase elastic composites: Comparisons and benchmarks. Technische Mechanik 30(4): 374-386.

Lielens G, Pirotte P, Couniot A, Dupret F and Keunings R (1998) Prediction of thermo-mechanical properties for compression moulded composites. Composites Part A: Applied Science and Manufacturing 29(1): 63-70.

Lubachevsky B and Stillinger F (1990) Geometric properties of random disk packings. Journal of statistical Physics 60(5-6): 561-583.

Lubachevsky B, Stillinger F and Pinson E (1991) Disks vs. spheres: Contrasting properties of random packings. Journal of Statistical Physics 64(3-4): 501-524.

Mori T and Tanaka K (1973) Average stress in matrix and average elastic energy of materials with misfitting inclusions. Acta metallurgica 21(5): 571-574.

Nakamura Y, Yamaguchi M, Okubo M and Matsumoto T (1992) Effects of particle size on mechanical and impact properties of epoxy resin filled with spherical silica. Journal of applied polymer science 45(7): 1281-1289.

Nemat-Nasser S and Hori M (2013) Micromechanics: overall properties of heterogeneous materials, volume 37. Elsevier.

Segurado J and Llorca J (2002) A numerical approximation to the elastic properties of sphere-reinforced composites. Journal of 
the Mechanics and Physics of Solids 50(10): 2107-2121.

Shi Y and Zhang Y (2006) Simulation of random packing of spherical particles with different size distributions. In: ASME 2006 International Mechanical Engineering Congress and Exposition. American Society of Mechanical Engineers, pp. 539-544.

Singh I, Shedbale A and Mishra B (2015) Material property evaluation of particle reinforced composites using finite element approach. Journal of Composite Materials 50(20): 2757-2771.

Spanoudakis J and Young R (1984) Crack propagation in a glass particle-filled epoxy resin - part 1 effect of particle volume fraction and size. Journal of Materials Science 19(2): 473-486.

Torquato S (1991) Random heterogeneous media: microstructure and improved bounds on effective properties. Applied mechanics reviews 44(2): 37-76.

Visscher W and Bolsterli M (1972) Random packing of equal and unequal spheres in two and three dimensions. Nature 239: 504507. 


\section{Tables}

\begin{tabular}{ccccc}
\hline Algorithm & Particle shape & Particles size distribution & Volume fraction & Max Volume fraction \\
\hline D\&R Rigid walls & circular & defined & calculated & 0.68 \\
D\&R Soft walls & circular & defined & calculated & 0.75 \\
Voronoi & polygonal & undefined & input of the model & 1 \\
D\&R Polygons & polygonal & defined & calculated & 0.60 \\
\hline
\end{tabular}

Table 1. Geometrical capacities of the microstructure

generation algorithms.

\begin{tabular}{ccc}
\hline Tensile 1-direction & Tensile 2-direction & Shear 12-direction \\
\hline$\underline{u_{1}}=\underline{U_{1}}$ & $\underline{u_{1}}=0$ & $\underline{u_{1}}=\underline{U_{2}}=\underline{U_{1}} / 2$ \\
$\underline{u_{2}}=0$ & $\underline{U_{2}} / 2$ \\
\hline
\end{tabular}

Table 2. Imposed displacements for the PBC, 3 different loading cases

\begin{tabular}{cccc}
\hline Phase & Mechanical symmetry & Young's modulus $E$ & Poisson's ratio $\nu$ \\
\hline Matrix & isotropic & $3 G P a$ & 0.3 \\
Particles & isotropic & $450 \mathrm{GPa}$ & 0.2 \\
\hline
\end{tabular}

Table 3. Material properties of the phases 


\section{Figures}

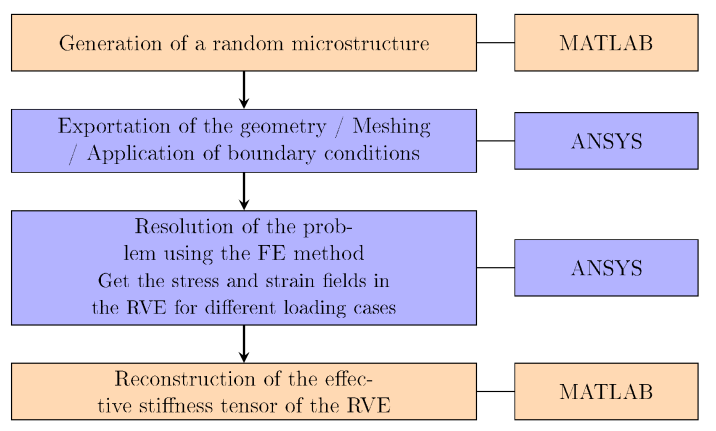

Figure 1. Numerical homogenisation scheme

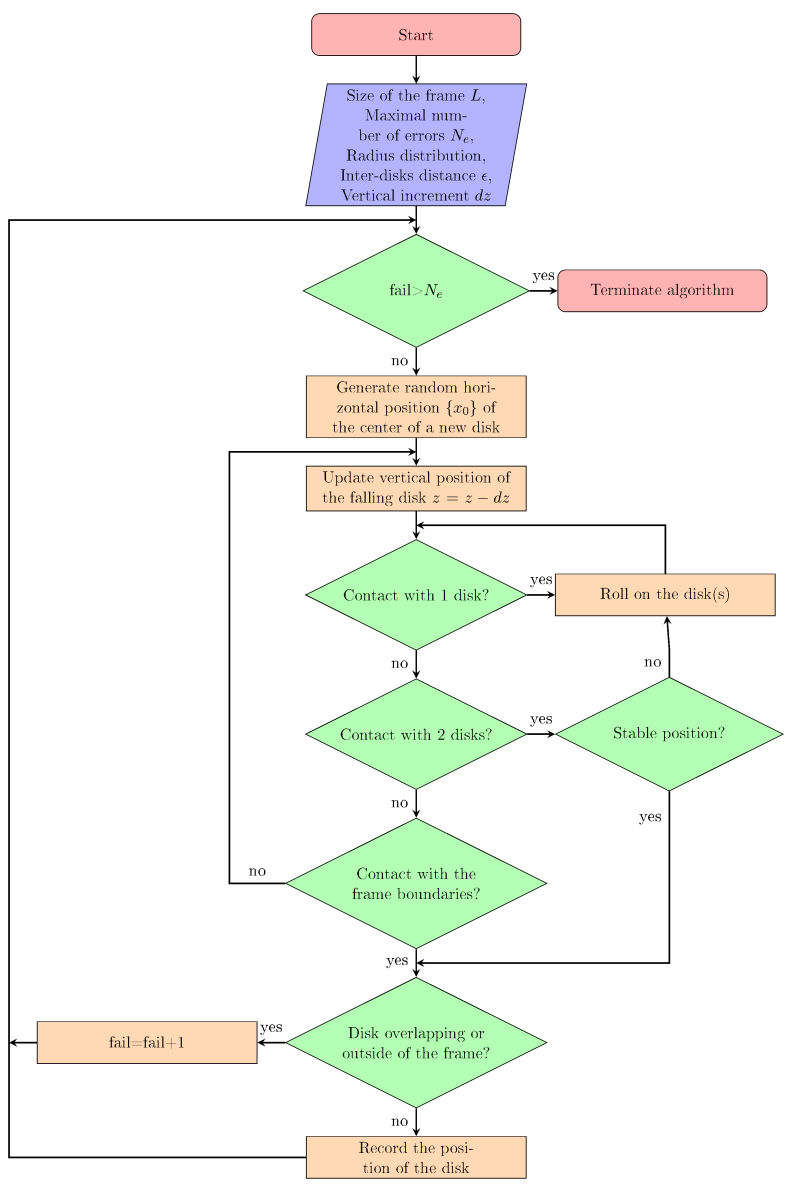

Figure 2. Flowchart of the dropping and rolling algorithm

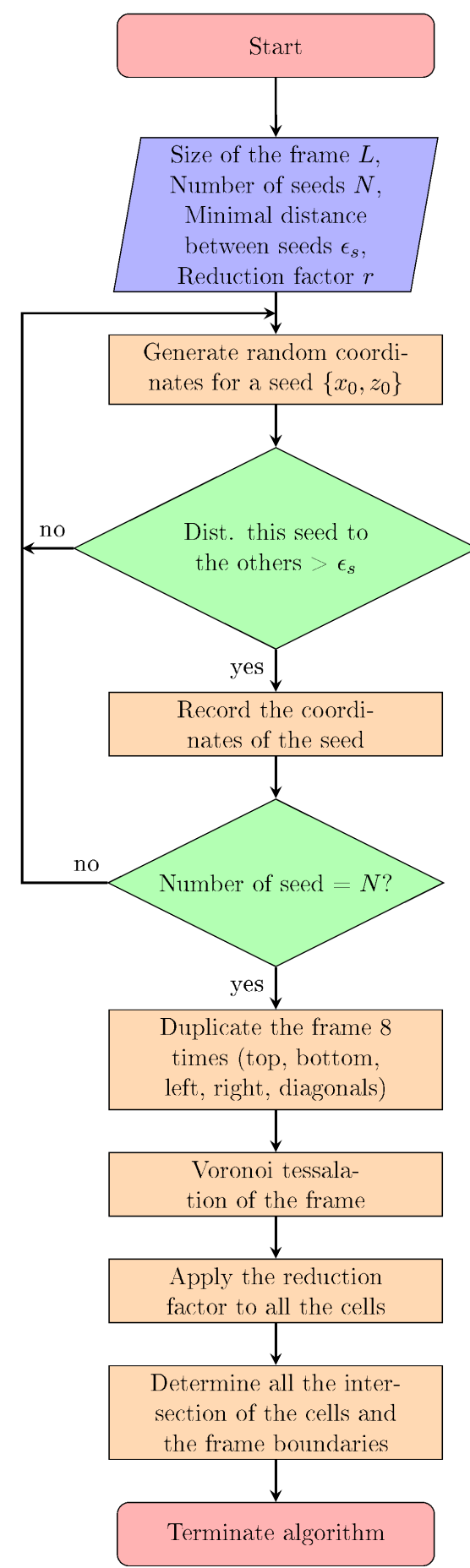

Figure 3. Flowchart of the Voronoi tesselation-based algorithm 


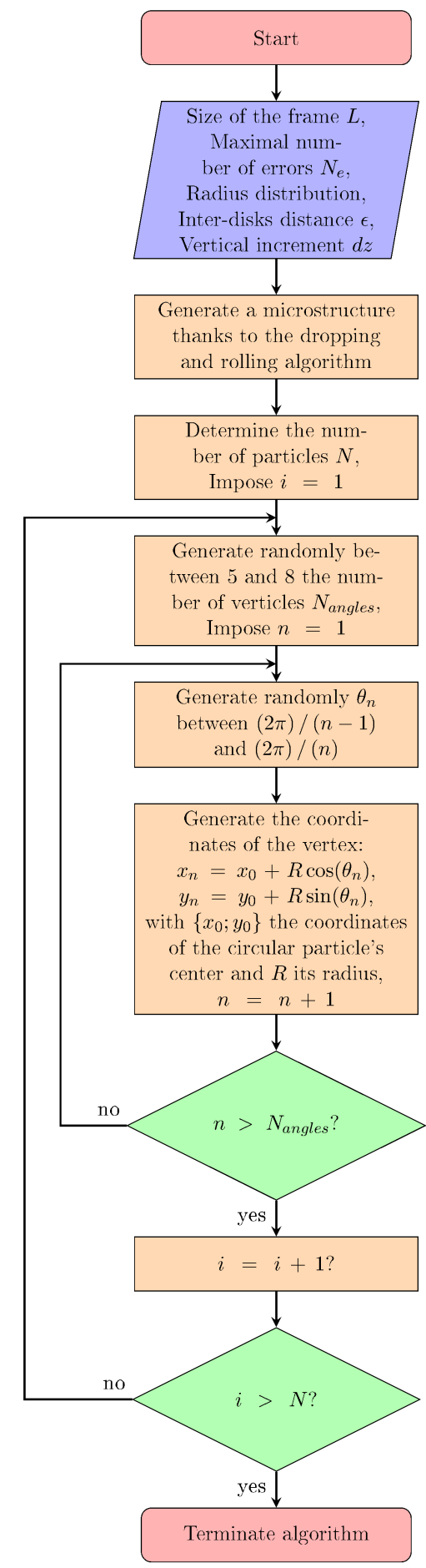

Figure 4. Flowchart of the dropping and rolling algorithm with polygonal particles

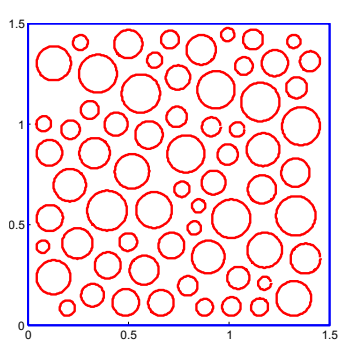

(a)

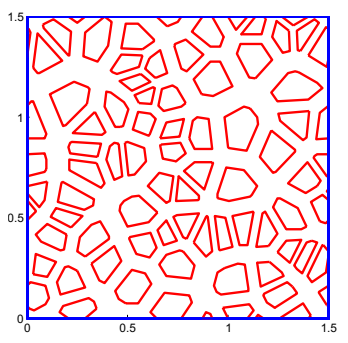

(c)

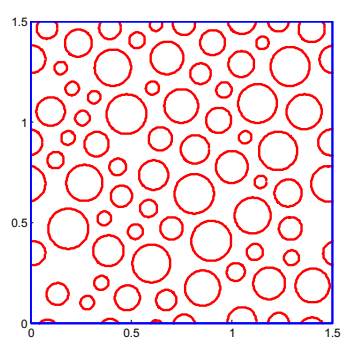

(b)

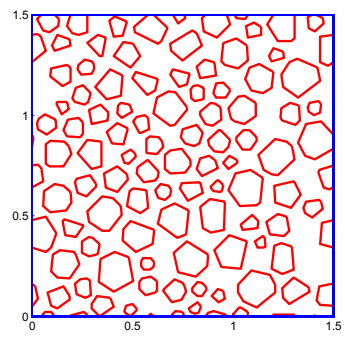

(d)
Figure 5. Microstructures obtained with the dropping and rolling algorithm with rigid walls (a), with soft walls (b), with Voronoi tesselation algorithm (c), with the dropping and rolling algorithm with polygonal particles (d). Volume fraction of particles for each microstructure $\phi=0.4$. 


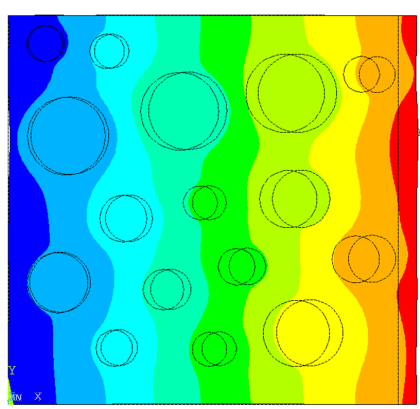

(a)

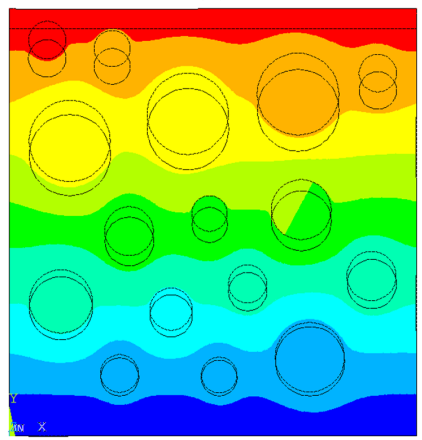

(b)

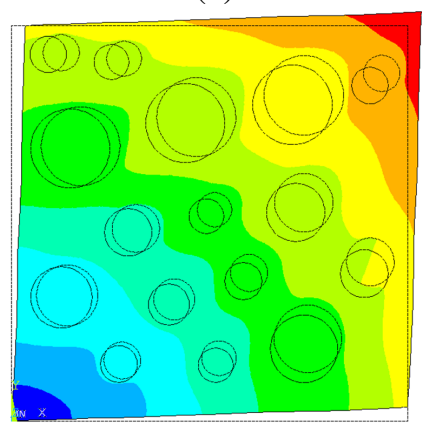

(c)

Figure 6. Displacement field $U_{11}$ of the RVE under tensile loading along 1-axis (a); displacement field $U_{22}$ of the RVE under tensile loading along 2-axis (b); displacement field $U_{12}$ of the RVE under shear loading in the 12-plane (c), [mm].

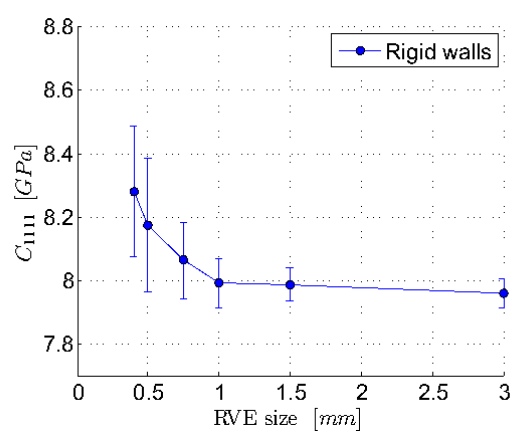

(a)

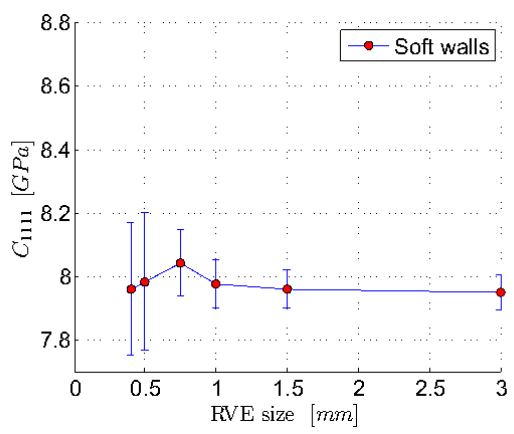

(b)

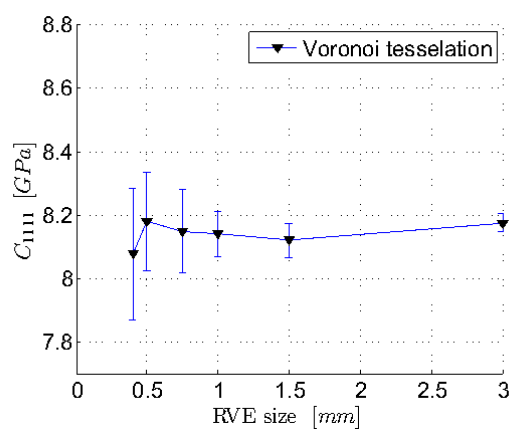

(c)

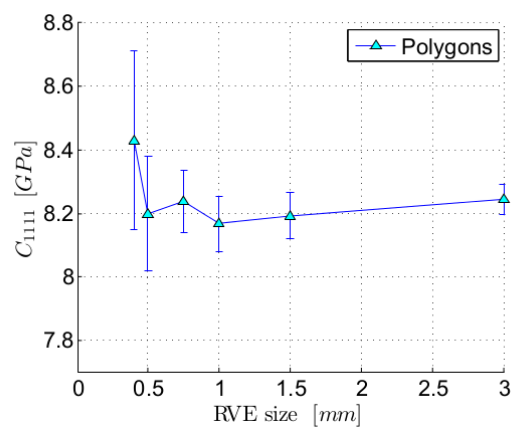

(d)

Figure 7. Evolution of the $C_{1111}$ component of the stiffness tensor with standard deviation against the size of the RVE for the dropping and rolling algorithm with rigid walls (a), with soft walls (b), for Voronoi tesselation algorithm (c), for the dropping and rolling algorithm with polygonal particles (d) for a fixed volume fraction $\phi=0.4$. 


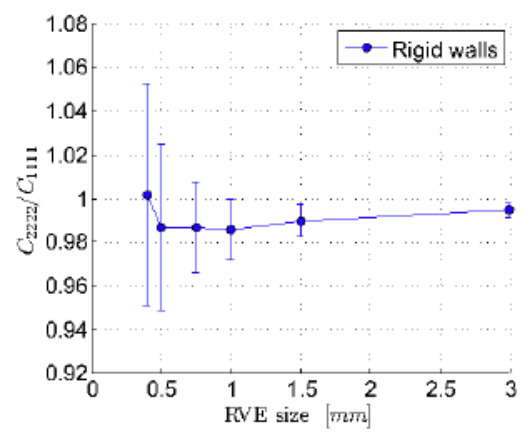

(a)

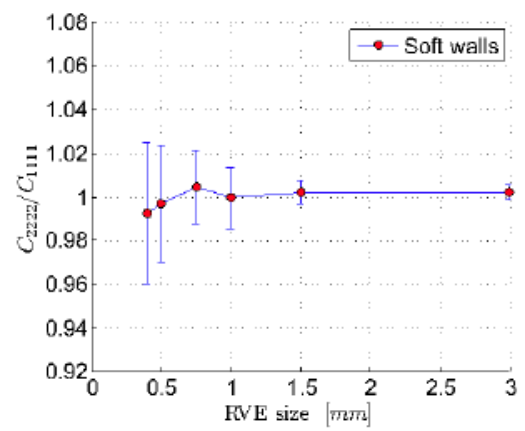

(b)

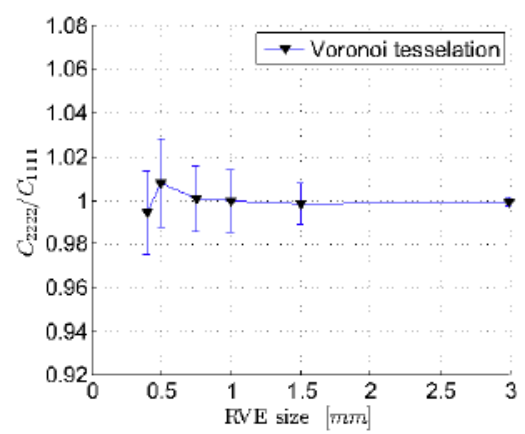

(c)

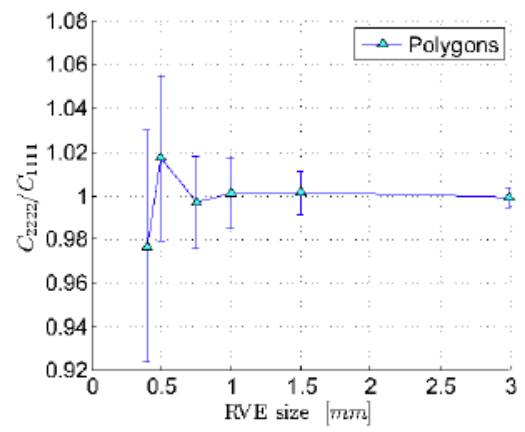

(c)

Figure 8. Evolution of the ratio $C_{2222} / C_{1111}$ with standard deviation against the size of the RVE for the dropping and rolling algorithm with rigid walls (a), with soft walls (b), for Voronoi tesselation algorithm (c), for the dropping and rolling algorithm with polygonal particles (d) for a fixed volume fraction $\phi=0.4$.

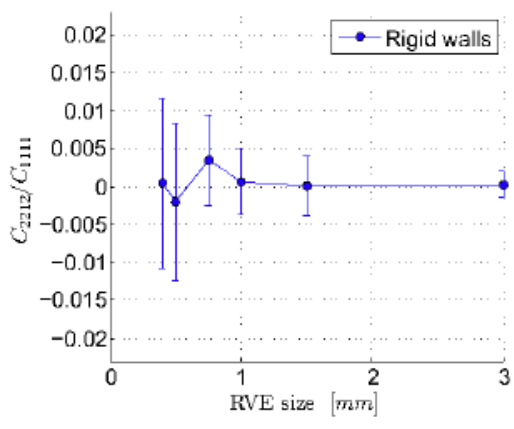

Figure 9. Evolution of the ratio $C_{2212} / C_{1111}$ with standard deviation against the size of the RVE for the dropping and rolling algorithm with rigid walls for a fixed volume fraction $\phi=0.4$.

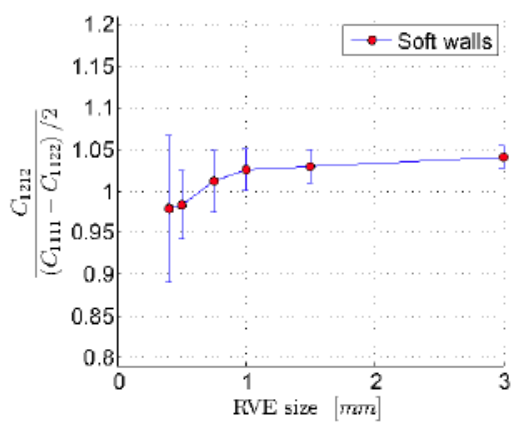

(a)

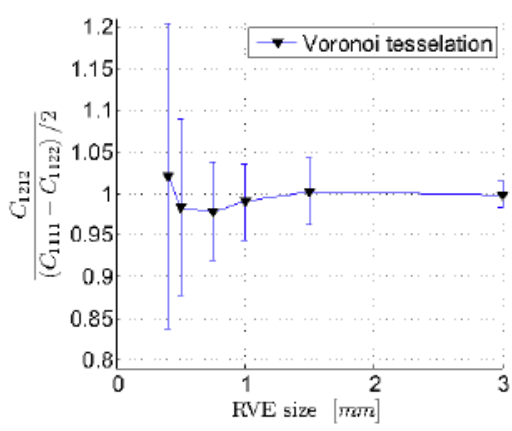

(b)

Figure 10. Evolution of the ratio $\left.C_{1212} /\left(\begin{array}{ll}C_{1111} & C_{1122}\end{array}\right) / 2\right)$ with standard deviation against the size of the RVE for the dropping and rolling algorithm with soft walls (a) and for Voronoi tesselation algorithm (b) for a fixed volume fraction $\phi=0.4$. 


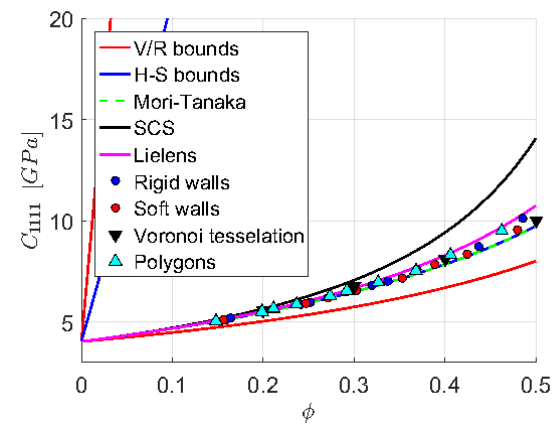

(a)

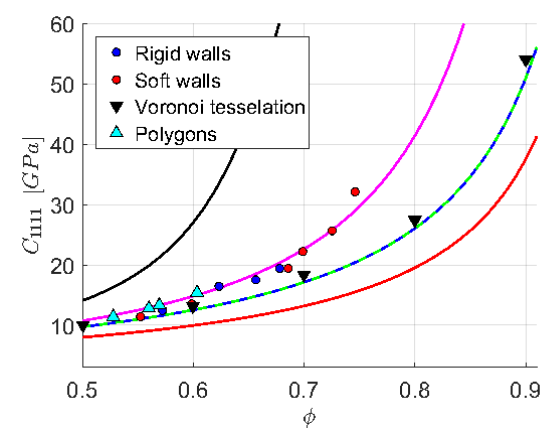

(b)

Figure 11. Homogenised $C_{1111}$ component of the stiffness tensor against volume fraction $\phi$ for analytical homogenisation schemes; V/R bounds $=$ Voigt and Reuss bounds, $\mathrm{H}$-S bounds = Hashin-Shtrikman bounds, Mori-Tanaka scheme, SCS = Self-Consistent Scheme, Lielens' model; and for numerical simulations on RVE for (a) low volume fractions (b) high volume fractions.

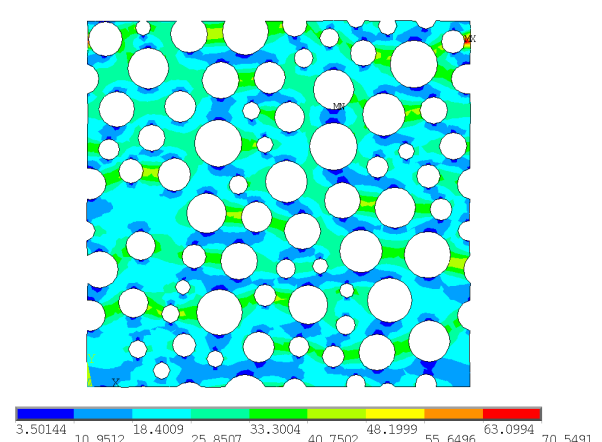

(a)

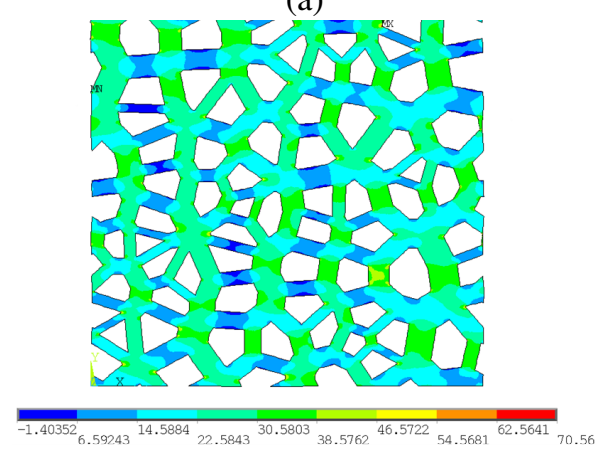

(b)

Figure 12. Local stress field $\sigma_{11}[\mathrm{MPa}]$ in the the matrix of the RVE for the dropping and rolling algorithm (a) and the Voronoi tesselation algorithm (b).

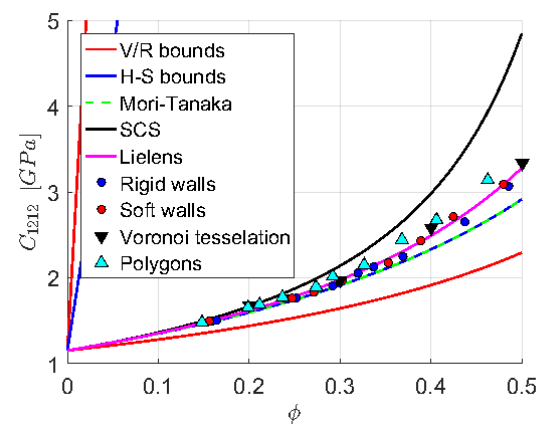

(a)

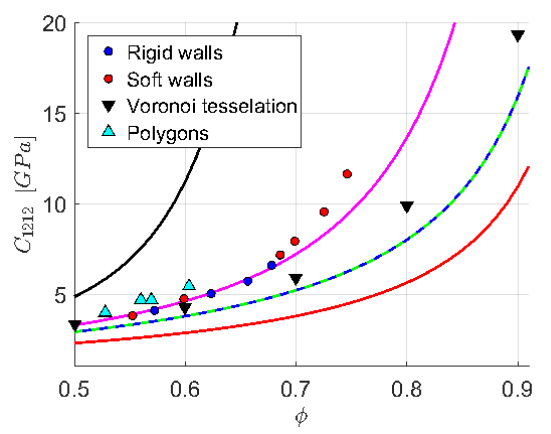

(b)

Figure 13. Homogenised $C_{1212}$ component of the stiffness tensor against volume fraction $\phi$ for analytical homogenisation schemes; V/R bounds = Voigt and Reuss bounds, $\mathrm{H}-\mathrm{S}$ bounds $=$ Hashin-Shtrikman bounds, Mori-Tanaka scheme, SCS = Self-Consistent Scheme, Lielens' model; and for numerical simulations on RVE for (a) low volume fractions (b) high volume fractions.

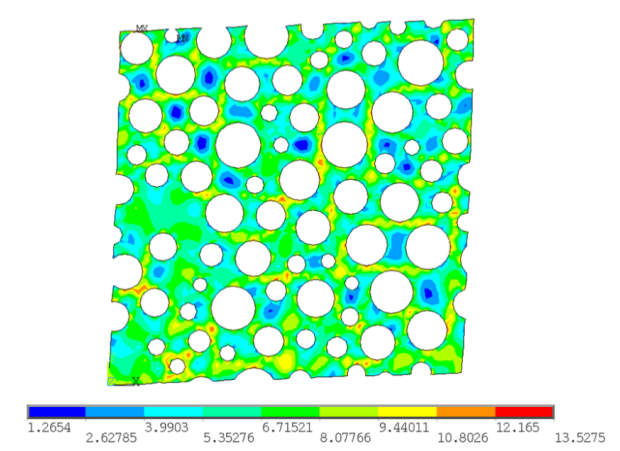

(a)

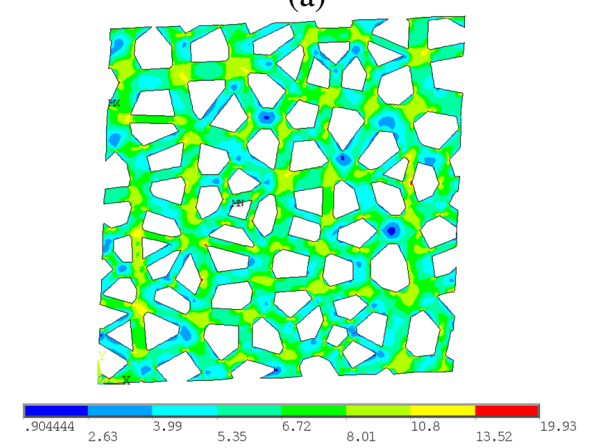

(b)

Figure 14. Local stress field $\sigma_{12}[\mathrm{MPa}]$ in the the matrix of the RVE for the dropping and rolling algorithm (a) and the Voronoi tesselation algorithm (b). 